\title{
Dissecting the dynamics of epigenetic changes in phenotype-structured populations exposed to fluctuating environments
}

\author{
Tommaso Lorenzi ${ }^{*, a, 1}$, Rebecca H. Chisholm ${ }^{\mathrm{b}, 1}$, Laurent Desvillettes ${ }^{\mathrm{a}}$, Barry D. Hughes ${ }^{\mathrm{c}}$ \\ ${ }^{a}$ Centre de Mathématiques et de Leurs Applications, ENS Cachan, CNRS, Cachan 94230 Cedex, France \\ ${ }^{b}$ School of Biotechnology and Biomolecular Sciences, University of New South Wales, Sydney NSW 2052, Australia \\ ${ }^{c}$ School of Mathematics and Statistics, University of Melbourne, Victoria 3010, Australia
}

\begin{abstract}
An enduring puzzle in evolutionary biology is to understand how individuals and populations adapt to fluctuating environments. Here we present an integro-differential model of adaptive dynamics in a phenotype-structured population whose fitness landscape evolves in time due to periodic environmental oscillations. The analytical tractability of our model allows for a systematic investigation of the relative contributions of heritable variations in gene expression, environmental changes and natural selection as drivers of phenotypic adaptation. We show that environmental fluctuations can induce the population to enter an unstable and fluctuation-driven epigenetic state. We demonstrate that this can trigger the emergence of oscillations in the size of the population, and we establish a full characterisation of such oscillations. Moreover, the results of our analyses provide a formal basis for the claim that higher rates of epimutations can bring about higher levels of intrapopulation heterogeneity, whilst intense selection pressures can deplete variation in the phenotypic pool of asexual populations. Finally, our work illustrates how the dynamics of the population size is led by a strong synergism between the rate of phenotypic variation and the frequency of environmental oscillations, and identifies possible ecological conditions that promote the maximisation of the population size in fluctuating environments.
\end{abstract}

Key words:

Phenotypic adaption, epimutations, environmental fluctuations, integro-differential equations, periodic solutions

\section{Introduction}

Evolution can be thought of as a complex and dynamic interplay between hereditary phenotypic modifications, environmental change and natural selection. In this framework, it is largely an open question in evolutionary biology how individuals and populations adapt to fluctuating environments.

Previous theoretical and experimental work involving asexual populations has shed some light on the way phenotypic diversity can evolve in the presence of environmental fluctuations [1]-[10]. With the aim of dissecting the relative contributions of phenotypic variation, environmental oscillations and natural selection as drivers of phenotypic adaptation, here we formulate and analyse an integrodifferential model of adaptive dynamics in a phenotypestructured population embedded in a changing environment. Models of this type can be derived from stochastic individual-based models in the limit of large numbers of individuals $[11,12]$, and they have been proven to constitute a suitable conceptual apparatus to study evolutionary processes in population dynamics [13]-[20].

\footnotetext{
*Corresponding author; E-mail address: lorenzi@cmla.enscachan.fr

${ }^{1}$ These primary authors contributed equally to this article
}

We focus on the ecological scenario where a population has a fitness landscape with one single peak, the location of which undergoes periodic oscillations in time. Due to random epimutation events (which change the way genes are expressed), individuals within this population undergo stochastic variation in phenotype [21]-[24]. We assume that small (large) epimutations correspond to small (large) phenotypic changes, and noting that small epimutations occur at a much higher frequency than large epimutations [25], we model the effects of heritable variations in gene expression by means of a diffusion operator, along the lines of Lorz et al. [26], Mirrahimi et al. [27], and Perthame et al. [28]. Moreover, in order to take into account the fact that epimutations can be inherently biased towards particular variants [33]-[36], we follow the modelling strategy presented in Chisholm et al. $[15,37]$ and include a drift operator in our model.

From the mathematical point of view, our work follows earlier papers on the analysis of integro-differential equations that arise in models of adaptive evolution of phenotype-structured populations [26]-[31]. These papers are devoted to the study of solutions of such equations when the rate of diffusion across the phenotypic space is small or tends to zero. The main novelty of our work is that we do not impose any smallness assumptions on the diffusion rate. We also allow the presence of a drift term 
in the governing equation. In this setting, we are able to establish the existence of periodic solutions with a Gaussian profile, without any specific assumptions concerning the nature of the periodic variation in the trait associated with the maximum of the fitness landscape.

Exploiting the analytical tractability of the model, we perform a systematic investigation of the ways in which the presence of a time-varying environment, the evolution of the epigenetic state, the level of phenotypic diversity and the size of the population are shaped by the rate of epimutations, the degree of bias in the generation of novel phenotypic variants, the strength of natural selection, and the frequency of environmental oscillations. The generality of this model makes the results of our study applicable to a broad range of asexual populations evolving in fluctuating environments.

\section{The model}

We study evolutionary dynamics in a well-mixed population that is structured by a phenotypic trait $x \in \mathbb{R}$. Individuals inside the population proliferate through asexual reproduction, die due to competition for limited resources, and undergo epimutations. To reduce biological complexity to its essence, we make the prima facie assumption that stochastic variations in gene expression yield infinitesimally small phenotypic modifications. Moreover, we let the environment evolve independently of the population [32]. Despite these simplifications, the model captures a wide spectrum of ecological scenarios.

The phenotype distribution of the population at time $t \geq 0$ is characterised by the population density $c(x, t) \geq 0$, which evolves through the following integro-differential equation:

$$
\frac{\partial c}{\partial t}+\alpha \frac{\partial c}{\partial x}=\beta \frac{\partial^{2} c}{\partial x^{2}}+R(x, t, \varrho(t)) c
$$

with

$$
\begin{gathered}
\varrho(t)=\int_{-\infty}^{\infty} c(x, t) d x, \\
c(x, t) \rightarrow 0 \text { as } x \rightarrow \pm \infty
\end{gathered}
$$

and

$$
c(x, 0) \in L^{1} \cap L^{\infty}(\mathbb{R}), \quad c(x, 0) \geq 0 \text { a.e. on } \mathbb{R} .
$$

It is natural to characterise the population's phenotype distribution in terms of its mean $\mu(t)$ and standard deviation $\sigma(t)$, given in the usual way by

$$
\begin{aligned}
\mu(t) & =\frac{1}{\varrho(t)} \int_{-\infty}^{\infty} x c(x, t) d x \\
\sigma^{2}(t) & =\frac{1}{\varrho(t)} \int_{-\infty}^{\infty} x^{2} c(x, t) d x-\mu(t)^{2} .
\end{aligned}
$$

If the distribution $c(x, t)$ is unimodal and reasonably symmetric, the mean phenotype will be close to the most prevalent phenotype, which has greater direct biological interest.

In Equation (2.1), the diffusion term models the effects of heritable variations in gene expression, which occur at the average rate $\beta \in \mathbb{R}_{+}$. The drift term accounts for the fact that epimutations can be more likely to produce phenotypic variations in certain directions, since the process leading to the introduction of novel phenotypic variants may be not purely random [33]-[36]. The sign of the parameter $\alpha \in \mathbb{R}$ models the direction of bias, and the absolute value of $\alpha$ measures the degree of bias. Natural selection is driven here by the fitness function $R(x, t, \varrho(t))$, which models the net proliferation rate of individuals with phenotypic trait $x$ at time $t$, given the total population size $\varrho(t)$. Throughout this paper, we make use of the following definition:

$$
R(x, t, \varrho(t)):=b(x, t)-\kappa \varrho(t) .
$$

The above definition relies on the idea that a higher total population corresponds to less available resources; therefore, we let individuals inside the population die at rate $\kappa \varrho(t)$, where the parameter $\kappa \in \mathbb{R}_{+}$models the average rate of death due to intrapopulation competition. Moreover, we let individuals with phenotypic trait $x$ at time $t$ proliferate or die at rate $b(x, t)$. Since we focus on a population with a single-peaked fitness landscape, we assume that the function $b$ is strictly concave in its first argument at each time instant $t$. In particular, we focus on the case where

$$
b(x, t):=\gamma-\epsilon[x-\varphi(t)]^{2},
$$

where $\gamma \in \mathbb{R}_{+}, \epsilon \in \mathbb{R}_{+}$and for some $T>0$

$$
\varphi:[0, \infty) \rightarrow \mathbb{R}, \quad \varphi(t)=\varphi(t+T) .
$$

The parameter $\gamma$ and the coefficient $\epsilon$ provide a measure of the strength of natural selection. Definition (2.9) mimics the effects of a fluctuating environment that induces the phenotypic trait associated with the maximum of the fitness landscape to change over time with period $T$.

\section{Analysis of the model}

Subject to a single condition below [the inequality (3.5)], there is a solution $c(x, t)=\mathcal{C}(x, t) \geq 0$ of the problem (2.1)-(2.4), where:

(i) $\mathcal{C}(x, t)$ is periodic with period $T$;

(ii) $\mathcal{C}(x, t)$ has a Gaussian profile,

$$
\mathcal{C}(x, t)=\frac{\varrho(t)}{\sqrt{\pi}}\left(\frac{\epsilon}{4 \beta}\right)^{1 / 4} \exp \left\{-\left(\frac{\epsilon}{4 \beta}\right)^{1 / 2}[x-\mu(t)]^{2}\right\},
$$

where $\mu(t)$ and $\varrho(t)$ are periodic (this ensures that the mean phenotype is also the most prevalent one); 
(iii) the instantaneous most prevalent phenotype $\mu(t)$ is

$$
\begin{aligned}
\mu(t)= & \frac{2(\epsilon \beta)^{1 / 2} e^{-2(\epsilon \beta)^{1 / 2} t}}{e^{2(\epsilon \beta)^{1 / 2} T}-1} \int_{0}^{T} e^{2(\epsilon \beta)^{1 / 2} \tau} \varphi(\tau) d \tau \\
& +2(\epsilon \beta)^{1 / 2} e^{-2(\epsilon \beta)^{1 / 2} t} \int_{0}^{t} e^{2(\epsilon \beta)^{1 / 2} \tau} \varphi(\tau) d \tau \\
& +\frac{\alpha}{2(\epsilon \beta)^{1 / 2}}
\end{aligned}
$$

and it oscillates with period $T$;

(iv) the mean (with respect to time) of the most prevalent phenotype is

$$
\bar{\mu}=\bar{\varphi}+\frac{\alpha}{2(\epsilon \beta)^{1 / 2}}
$$

with

$$
\bar{\varphi}=\frac{1}{T} \int_{0}^{T} \varphi(t) d t
$$

(v) the instantaneous total population $\varrho(t)$ oscillates

- with period $T / 2$ in the case when $\alpha=0$ and $\varphi(t+T / 2)=-\varphi(t)$,

- with period $T$ in all other cases,

and it is given by

$$
\varrho(t)=\frac{\kappa^{-1} \exp \left[\int_{0}^{t} Q(\tau) d \tau\right]}{r+\int_{0}^{t} \exp \left[\int_{0}^{\xi} Q(\tau) d \tau\right] d \xi},
$$

with

$$
r=\frac{\int_{0}^{T} \exp \left[\int_{0}^{\xi} Q(\tau) d \tau\right] d \xi}{\exp \left[\int_{0}^{T} Q(\tau) d \tau\right]-1}
$$

and

$$
Q(t)=\gamma-\epsilon[\varphi(t)-\mu(t)]^{2}-(\epsilon \beta)^{1 / 2}
$$

(vi) the mean (with respect to time) of the total population is

$$
\bar{\varrho}=\frac{1}{\kappa}\left\{\gamma-(\epsilon \beta)^{1 / 2}-\frac{\epsilon}{T} \int_{0}^{T}[\varphi(t)-\mu(t)]^{2} d t\right\} .
$$

For these conclusions to hold, the only additional restriction on the parameters $\alpha, \beta, \gamma$ and $\epsilon$ and the function $\varphi(t)$ is that

$$
\gamma-(\epsilon \beta)^{1 / 2}-\frac{\epsilon}{T} \int_{0}^{T}[\varphi(t)-\mu(t)]^{2} d t>0,
$$

corresponding to $\bar{\varrho}>0$.

The proofs of (i)-(vi) are detailed in Appendix A. These results are illustrated by means of numerical solutions in Section 4, and they convey the following pieces of ecological information. (i) Oscillations with period $T$ in the location of the fitness maximum induce oscillations with the same period in the phenotype distribution $c(x, t)$.

(ii) The oscillating phenotype distribution is always unimodal, and the mean phenotype is always close to the most prevalent phenotype. The measure of phenotypic diversity $\sigma^{2}(t)$ remains constant in time and satisfies

$$
\sigma^{2}(t)=\sqrt{\frac{\beta}{\epsilon}} \quad \text { for all } t>0
$$

i.e, phenotypic diversity increases with the rate of epimutations $\beta$ and decreases with the strength of natural selection $\epsilon$.

(iii) The most prevalent phenotype $\mu(t)$ oscillates with period $T$.

(iv) A bias in the generation of novel phenotypic variants shifts the time average $\bar{\mu}$ of the most prevalent phenotype away from the time average $\bar{\varphi}$ of the trait associated with the maximum of the fitness landscape.

(v) The total population $\varrho(t)$ oscillates with period $T$ except under some some special restrictions (absence of bias in the generation of novel phenotypic variants and a certain temporal symmetry in the periodic environmental fluctuations) when the total population can oscillate with period $T / 2$.

(vi) The time average $\bar{\varrho}$ of the global population density depends in a nonlinear way on the period of oscillations in the fittest phenotype $T$, the rate of epimutations $\beta$, and the strength of natural selection $\epsilon$. The explicit dependence of $\bar{\varrho}$ on the mean of the squared distance between $\mu(t)$ and the fittest phenotype $\varphi(t)$ also ensures its dependence on the drift parameter $\alpha$.

Remark 1. Numerical simulations (data not shown), suggest that analogous results hold in the case where, instead of considering epimutations through a differential operator, an integral operator is used to model the effects of genetic variations, on the condition that the mutation kernel is Gaussian and the related variance is sufficiently small.

\section{Numerical solutions}

In order to illustrate the analytical results established in the previous section, here we present the results of numerical solutions for the mathematical problem (2.1)-(2.4) with $t \in[0 ; 4 T], x \in[-L ; L]$ and

$$
\varphi(t):=\frac{L}{2} \sin (\omega t), \quad \omega=\frac{2 \pi}{T} .
$$

Further technical details of the numerical solution method are provided in Appendix B, but we mention here several important general points. The model that we have 
analysed in full mathematical detail is defined with $x \in$ $(-\infty, \infty)$, but the finite-interval numerical solutions exhibit the same qualitative behaviour. In more detail, the time average of the most prevalent phenotype and the time average of the total population found by our numerical procedure match closely the values predicted by (iv) and (vi) once a very short interval of transient behaviour has finished. Although the initial total population chosen in our numerical computations is significantly different from the time-averaged mean population in the periodic solution, the time taken for clear periodic behaviour with the correct mean population averaged over a period to emerge is short.

\subsection{Environmental fluctuations lead to oscillations in the phenotypic distribution, in the dominant phenotype, and in the total population}

We are considering an ecological scenario where the population has a fitness landscape with one single global maximum, whose location undergoes periodic oscillations due to the effects of environmental fluctuations. Accordingly, the results established by (i),(ii),(iii),(v) and illustrated in Figure 1(A) show that, independently from the profile of the phenotypic distribution at the beginning of observations (i.e., the initial condition $c^{0}(x)$ ), the population quickly becomes unimodal. Moreover, as is highlighted by Figure 1(A),(C),(D) and Figure 2, the $T$-periodic oscillations of the trait associated with the maximum of the fitness landscape $\varphi(t)$ cause the emergence of oscillations in the phenotypic distribution $c(x, t)$, in the most prevalent phenotype $\mu(t)$, and in the total population $\varrho(t)$.

Both the phenotypic distribution and the dominant phenotype oscillate in time with period $T$. On the other hand, with the exception of an initial boundary-layer, the total population oscillates with period $T / 2$ when there is no bias in the generation of novel phenotypic variants (i.e., when $\alpha=0$ ), and with period $T$ in the presence of bias (i.e., when $\alpha \neq 0$ ). The degree of bias $|\alpha|$ affects both the profile and the amplitude of the oscillations in the total population (see Figure 2(B)), while it leaves unaltered the oscillations in the most prevalent phenotype (see Figure 2(A)).

\subsection{The level of phenotypic diversity increases with the rate of epimutations and decreases with the strength of natural selection}

The result established by (ii) and illustrated in Figure 1(B) demonstrates that the level of phenotypic diversity $\sigma^{2}$ remains constant in time and it is not altered by environmental fluctuations. Moreover, the phase diagram presented in Figure 3 illustrates how the level of phenotypic diversity is affected by the rate of epimutations $\beta$ and the strength of natural selection $\epsilon$. In summary, $\sigma^{2}$ increases with $\beta$ and decreases with $\epsilon$. Therefore, as one would expect, phenotypic diversity is brought about by frequent epimutations and it is curtailed by stronger selection pressures.
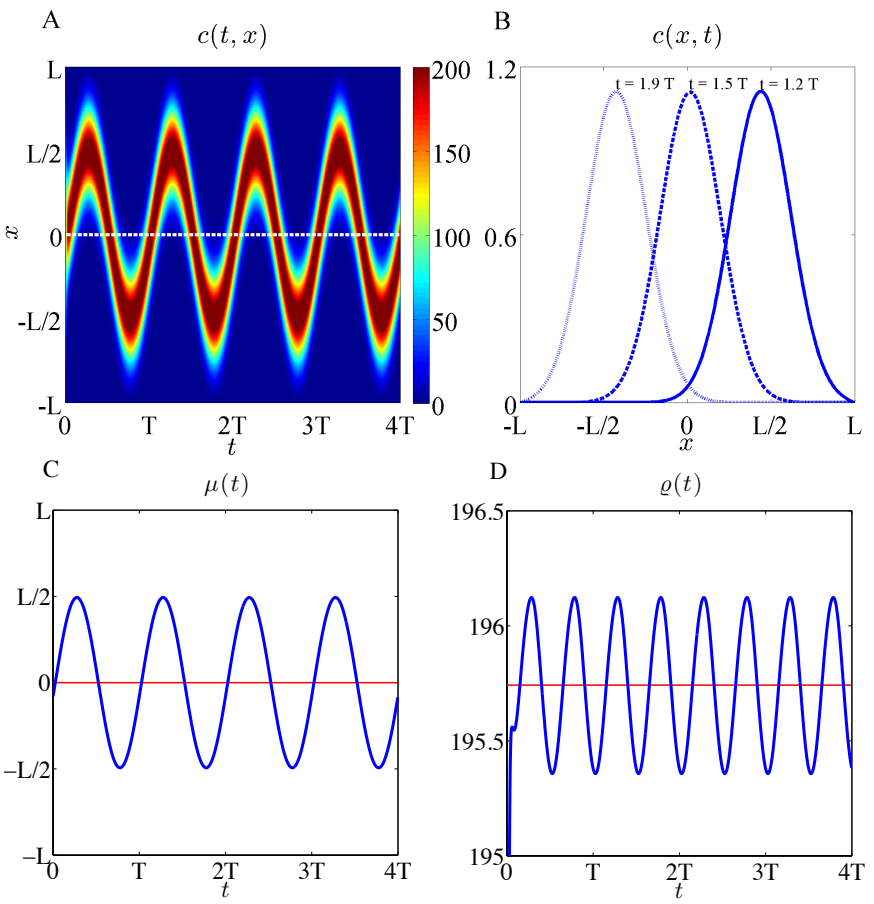

Figure 1: Environmental fluctuations cause the emergence of oscillations in the phenotypic distribution, in the most prevalent phenotype, and in the total population. (A) Plot of the phenotypic distribution $c(t, x)$ for $t \in[0 ; 4 T]$. The white line corresponds to the time average of the most prevalent phenotype $\bar{\mu}$ from (iv). (B) Plots of the phenotypic distribution $c(x, t)$ at $t \approx 1.2 T$ (solid line), $t \approx 1.5 T$ (dashed line) and $t \approx 1.9 T$ (dotted line). (C) Plot of the most prevalent phenotype $\mu(t)$ for $t \in[0 ; 4 T]$. The value of the time average $\bar{\mu}$ from (iv) is highlighted by the red line. (D) Plot of the total population $\varrho(t)$ for $t \in[0 ; 4 T]$. The red line corresponds to the time average $\bar{\varrho}$ from (vi). Further technical details of the numerical solutions are provided in Appendix B.
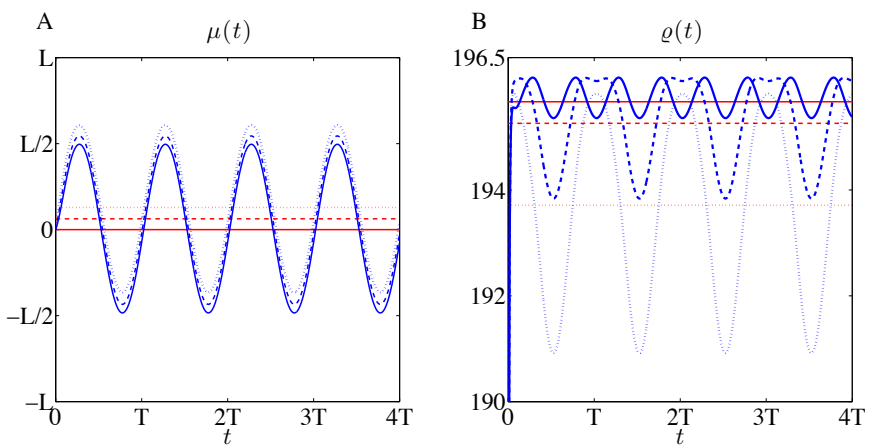

Figure 2: The period of oscillations in the total population depends on the degree of bias in the generation of novel phenotypic variants. (A) Plot of the most prevalent phenotype $\mu(t)$ for $t \in[0 ; 4 T]$. (B) Plot of the total population $\varrho(t)$ for $t \in[0 ; 4 T]$. The red lines highlight the time averages of the most prevalent phenotype $\bar{\mu}$ and total population $\bar{\varrho}$ from (iv) and (vi), respectively. Solid, dashed and dotted lines correspond to increasing degrees of bias in the generation of novel phenotypic variants, that is, $\alpha=0$ (solid lines), $\alpha=1$ (dashed lines) and $\alpha=2$ (dotted lines). Further technical details of the numerical solutions are provided in Appendix B. 


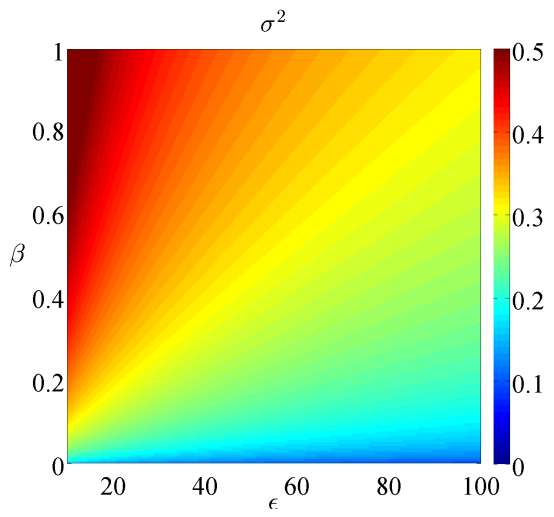

Figure 3: The level of phenotypic diversity depends on the rate of epimutations and the strength of natural selection. Plot of the level of phenotypic diversity $\sigma^{2}$ as a function of the strength of natural selection $\epsilon$ and the rate of epimutations $\beta$. Further technical details of the numerical solutions are provided in Appendix B.

\subsection{A bias in the generation of novel phenotypic variants paves the way for suboptimal adaptation}

The result established by (iv) suggests that a bias in the generation of novel phenotypic variants paves the way for suboptimal adaptation by shifting the time average of the most prevalent phenotype $\bar{\mu}$ away from the time average of the fittest phenotype $\bar{\varphi}$ (i.e., the distance $|\bar{\mu}-\bar{\varphi}|$ is different from zero when $\alpha \neq 0$ ). This is illustrated by the phase diagrams in Figure 4, which show that higher degrees of bias $|\alpha|$ correspond to larger deviations in $\bar{\mu}$ from $\bar{\varphi}$. Furthermore, the result established by (iv) and illustrated in Figure 4 demonstrates that increasing either the strength of natural selection $\epsilon$ or the rate of epimutations $\beta$ acts to reduce the distance $|\bar{\mu}-\bar{\varphi}|$. Taken together, these results support the idea that, while a bias in the generation of novel phenotypic variants can cause suboptimal adaptation, strong natural selection and frequent fluctuations in gene expression can reduce the deviation of the time average of the dominant phenotype in the population from the time average of the most adapted phenotype.

\subsection{The time average of the population size depends on a complex interplay between the rate of phenotypic vari- ation and the frequency of environmental fluctuations}

The result established by (vi) demonstrates that the mean (with respect to time) of the total population $\bar{\varrho}$ is the result of a complex interplay between the period $T$ of the oscillations in the trait associated with the maximum of the fitness landscape, the rate of epimutations $\beta$, the strength of natural selection $\epsilon$, and the time average of the square of the distance between the most prevalent phenotype $\mu(t)$ and the fittest phenotype $\varphi(t)$.

In order to shed some light on the ecological conditions that favour the maximisation of the average size of asexual populations in fluctuating environments, we investigate the values of the epimutation rate that correspond to higher values of the average total population, and
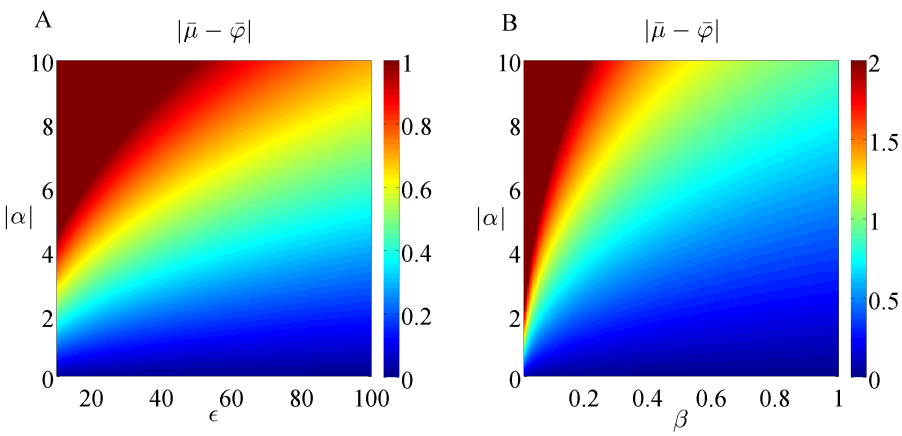

Figure 4: A bias in the generation of novel phenotypic variants paves the way for suboptimal adaptation. (A) Plot of the distance between the time average of the most prevalent phenotype $\bar{\mu}$ and the time average of the fittest phenotype $\bar{\varphi}$ as a function of the strength of natural selection $\epsilon$ and the degree of bias in the generation of novel phenotypic variants $\alpha$. (B) Plot of the distance between the time average of the most prevalent phenotype $\bar{\mu}$ and the time average of the fittest phenotype $\bar{\varphi}$ as a function of the rate of epimutations $\beta$ and the degree of bias in the generation of novel phenotypic variants $\alpha$. Further technical details of the numerical solutions are provided in Appendix B.

whether these values vary with the period of environmental fluctuations. To this end, we construct numerical solutions while holding all parameters constant except for $T$ and $\beta$, and we record the resulting values of $\bar{\varrho}$.

The results obtained are summarised in the phase diagram of Figure 5, which shows how the mean of the total population varies as a function of the period of environmental oscillations and the rate of epimutations. In more detail, when the environment changes rarely (i.e., for large values of $T$ ), the larger values of the average total population are attained for low values of the epimutation rate. On the other hand, if environmental oscillations are moderately fast (i.e., for intermediate values of $T$ ), higher epimutation rates correspond to larger average total populations. Finally, in the case of fast environmental fluctuations (i.e., for low values of $T$ ), the mean of the total population is maximised by some very low values of the epimutation rate.

\section{Discussion and conclusions}

Recently, Serviedio and co-workers observed that an important purpose of mathematical models in evolutionary research is "to act as 'proof-of-concept' tests of the logic in verbal explanations, paralleling the way in which empirical data are used to test hypotheses" [38]. In this spirit, our goal here is to contribute to a systematic identification of the relative contributions of heritable variations in gene expression, environmental changes and natural selection as drivers of adaptation in populations that evolve in fluctuating environments. To this end, we have presented an integro-differential model of adaptive dynamics in a population that is genetically uniform, but structured with respect to a phenotypic trait. 


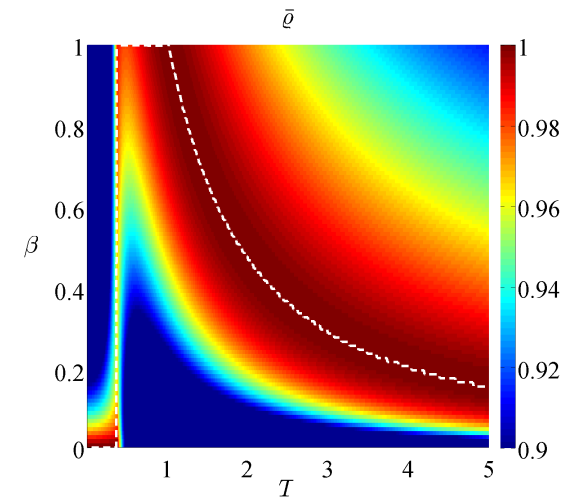

Figure 5: The time average of the population size depends on a complex interplay between the rate of epimutations and the frequency of environmental fluctuations. Plot of the time average of the total population $\bar{\varrho}$ as a function of the period of environmental fluctuations $T \in[0 ; 5]$ and the rate of epimutations $\beta \in[0 ; 1]$. For each $T$, the value of $\bar{\varrho}$ is normalised with respect to $\max _{\beta} \bar{\varrho}(T, \beta)$. The white line highlights the value of $\arg \max _{\beta} \bar{\varrho}(T, \beta)$ with $\bar{\varrho}$ given by (vi). Further technical details of the numerical solutions are provided in Appendix B.

Focusing on the case where the trait associated with the maximum of the fitness landscape fluctuates in time due to periodic environmental changes, we have shown that environmental oscillations can induce the population to enter an unstable and fluctuation-driven epigenetic state. We have also demonstrated that this can trigger the emergence of oscillations in the size of the population. Moreover, we have traced out a possible connection between the frequency of the oscillations and the presence of a bias in the introduction of novel phenotypic variants, which suggests a possible indirect way to quantitatively test and validate (or dispute) existing hypotheses about bias-led evolution. In this respect, an experimental setting analogous to that presented in Acar et al. [1] — which relies on the use of isogenic populations that evolve in laboratory-based fluctuating environments - may prove to be useful.

A large body of evidence indicates that transcription regulation is inherently stochastic at various levels and can give rise to significant phenotypic heterogeneity within asexual populations [21]-[24]. Our study provides some insight into the way in which the level of phenotypic diversity depends on the strength of natural selection and the rate of heritable variations in gene expression. Specifically, the results of our analysis formalise the idea that higher rates of epimutations lead to higher levels of intrapopulation heterogeneity, whilst intense selection pressures can deplete variation in the phenotypic pool of asexual populations, thus causing less phenotypic diversity. This is consistent with the experimental results presented in [39], where it was found that a more gradual increase in ambient $\mathrm{CO}_{2}$ concentration resulted in a substantially higher species richness in a mycorrhizal fungi community, in comparison to the abrupt change typical of other $\mathrm{CO}_{2}$ elevation experiments.
It might be speculated that because epimutation rates are small in comparison to selection coefficients, the direction of evolution is determined exclusively by natural selection. On the contrary, here we have shown that a bias in the introduction of novel phenotypic variants can strongly influence the course of phenotypic adaptation, even when the epimutation rate is small in comparison to the strength of natural selection [40]. Our results indicate that epimutational bias can pave the way for suboptimal adaptation by inducing a shift between the more prevalent phenotypes in a population and the phenotypes that are more successfully adapted to the surrounding environment. This effect escalates as the degree of bias increases, but it can be offset by strong natural selection and frequent fluctuations in gene expression.

Our analytical work provides evidence that the value of the time average of the population size results from a strong synergism between the period of environmental oscillations and the rate of epimutations. Furthermore, the results of our numerical solutions testify to the idea that the mean size of the population is maximised by low rates of phenotypic variation when the environment changes rarely, whereas higher rates of epimutation promote a larger average population size in the presence of moderately-fast environmental fluctuations. These findings recapitulate, to an extent, the results presented in previous theoretical and experimental work [1]-[10], and provide evidence for the importance of stochastic phenotypic switching as a mechanism for coping with changing environments. Finally, in the presence of fast environmental oscillations, the maximisation of the mean population size is achieved through low rates of phenotypic variation. In agreement with previous studies of phenotypic plasticity $[41,42]$, this result suggests that, in the case where environmental changes are highly unpredictable, high rates of epimutation can increase rather than decrease the risk of extinction.

Accordingly, we predict that environments which fluctuate slowly favour individuals with low rates of stochastic phenotypic variation. On the other hand, environments that fluctuate more rapidly favour those individuals that are endowed with high rates of phenotypic variation. However, if the rate of environmental fluctuations keeps increasing, individuals with very low rates of phenotypic variation will eventually revert to being the most competitive in the struggle for survival.

\section{Acknowledgments}

This work was supported in part by the French National Research Agency through the "ANR blanche" project Kibord [ANR-13-BS01-0004] and by the Australian Research Council [DP110100795 and DP140100339]. TL was also supported by the Hadamard Mathematics Labex, backed by the Fondation Mathématique Jacques Hadamard, through a grant overseen by the French National Research Agency [ANR-11-LABX-0056-LMH]. 


\section{A. Proof of (i)-(vi)}

In this appendix we prove the results (i)-(vi).

\section{A.1. The differential equations for $\mu(t)$ and $\varrho(t)$}

Since

$\log [\mathcal{C}(x, t)]=\log [\varrho(t)]-\left(\frac{\epsilon}{4 \beta}\right)^{1 / 2}[x-\mu(t)]^{2}+$ constant

it follows that

$$
\begin{aligned}
\frac{1}{\mathcal{C}} \frac{\partial \mathcal{C}}{\partial t} & =\frac{\varrho^{\prime}(t)}{\varrho(t)}+\left(\frac{\epsilon}{\beta}\right)^{1 / 2}[x-\mu(t)] \mu^{\prime}(t), \\
\frac{1}{\mathcal{C}} \frac{\partial \mathcal{C}}{\partial x} & =-\left(\frac{\epsilon}{\beta}\right)^{1 / 2}[x-\mu(t)], \\
\frac{1}{\mathcal{C}} \frac{\partial^{2} \mathcal{C}}{\partial x^{2}} & =-\left(\frac{\epsilon}{\beta}\right)^{1 / 2}+\frac{\epsilon}{\beta}[x-\mu(t)]^{2},
\end{aligned}
$$

and so inserting $c(x, t)=\mathcal{C}(x, t)$ in Eq. (2.1) as a trial solution we find that we require the following equation to hold as an identity:

$$
\begin{array}{r}
\frac{\varrho^{\prime}(t)}{\varrho(t)}+\left(\frac{\epsilon}{\beta}\right)^{1 / 2}[x-\mu(t)] \mu^{\prime}(t)-\alpha\left(\frac{\epsilon}{\beta}\right)^{1 / 2}[x-\mu(t)] \\
=\beta\left\{-\left(\frac{\epsilon}{\beta}\right)^{1 / 2}+\frac{\epsilon}{\beta}[x-\mu(t)]^{2}\right\} \\
+\gamma-\epsilon[x-\varphi(t)]^{2}-\kappa \varrho(t) .
\end{array}
$$

If we expand both sides in powers of $x$, then the coefficients of the terms in $x^{2}$ match correctly (the trial solution was chosen to achieve this), while the coefficients of $x^{1}$ and $x^{0}$, respectively, give us two differential equations:

$$
\begin{aligned}
\mu^{\prime}(t)-\alpha= & -2(\epsilon \beta)^{1 / 2} \mu(t)+2(\epsilon \beta)^{1 / 2} \varphi(t) \\
\frac{\varrho^{\prime}(t)}{\varrho(t)}= & \left(\frac{\epsilon}{\beta}\right)^{1 / 2}\left[\mu(t) \mu^{\prime}(t)-\alpha \mu(t)\right]-(\epsilon \beta)^{1 / 2} \\
& +\epsilon \mu(t)^{2}+\gamma-\epsilon \varphi(t)^{2}-\kappa \varrho(t)
\end{aligned}
$$

We can tidy up these equations. Equation (A.1) can be rewritten as a first-order linear equation,

$$
\mu^{\prime}(t)+2(\epsilon \beta)^{1 / 2} \mu(t)=\alpha+2(\epsilon \beta)^{1 / 2} \varphi(t),
$$

while on eliminating $\mu^{\prime}(t)$ from Eq. (A.2) using Eq. (A.1), we find that

$$
\frac{\varrho^{\prime}(t)}{\varrho(t)}=Q(t)-\kappa \varrho(t)
$$

where for brevity we have written

$$
Q(t)=\gamma-\epsilon[\varphi(t)-\mu(t)]^{2}-(\epsilon \beta)^{1 / 2} .
$$

\section{A.2. Mean values of $\mu(t)$ and $\varrho(t)$ if they are periodic}

Before we investigate whether these differential equations have any solutions with the same periodicity as $\varphi(t)$, we shall find what the mean values

$$
\bar{\mu}=\frac{1}{T} \int_{0}^{T} \mu(t) d t, \quad \bar{\varrho}=\frac{1}{T} \int_{0}^{T} \varrho(t) d t,
$$

of $\mu(t)$ and $\varrho(t)$, respectively, over a period would have to be.

Integrating Eq. (A.3) over $[0, T]$, dividing by $T$ and requiring $\mu(0)=\mu(T)$ leads to

$$
\bar{\mu}=\bar{\varphi}+\frac{\alpha}{2(\epsilon \beta)^{1 / 2}} .
$$

Similarly, integrating Eq. (A.4) over $[0, T]$, dividing by $T$ and requiring $\varrho(0)=\varrho(T)$ leads to

$$
\begin{aligned}
\bar{\varrho} & =\frac{\bar{Q}}{\kappa}=\frac{1}{\kappa T} \int_{0}^{T} Q(t) d t \\
& =\frac{1}{\kappa}\left\{\gamma-(\epsilon \beta)^{1 / 2}-\frac{\epsilon}{T} \int_{0}^{T}[\varphi(t)-\mu(t)]^{2} d t\right\} .
\end{aligned}
$$

\section{A.3. A periodic solution for $\mu(t)$}

Using the usual integrating factor technique for linear ordinary differential equations of first order, we find that

$$
\begin{aligned}
& \frac{d}{d t}\left[\mu(t) \exp \left[2(\epsilon \beta)^{1 / 2} t\right]\right\} \\
& \quad=\alpha \exp \left[2(\epsilon \beta)^{1 / 2} t\right]+2(\epsilon \beta)^{1 / 2} \exp \left[2(\epsilon \beta)^{1 / 2} t\right] \varphi(t)
\end{aligned}
$$

and so

$$
\begin{aligned}
& \mu(t) \exp \left[2(\epsilon \beta)^{1 / 2} t\right]-\mu(0) \\
& =\frac{\alpha}{2(\epsilon \beta)^{1 / 2}}\left\{\exp \left[2(\epsilon \beta)^{1 / 2} t\right]-1\right\} \\
& \quad+2(\epsilon \beta)^{1 / 2} \int_{0}^{t} \exp \left[2(\epsilon \beta)^{1 / 2} \tau\right] \varphi(\tau) d \tau,
\end{aligned}
$$

from which it follows that

$$
\begin{aligned}
\mu(t) & =\left[\mu(0)-\frac{\alpha}{2(\epsilon \beta)^{1 / 2}}\right] \exp \left[-2(\epsilon \beta)^{1 / 2} t\right]+\frac{\alpha}{2(\epsilon \beta)^{1 / 2}} \\
& +2(\epsilon \beta)^{1 / 2} \exp \left[-2(\epsilon \beta)^{1 / 2} t\right] \int_{0}^{t} \exp \left[2(\epsilon \beta)^{1 / 2} \tau\right] \varphi(\tau) d \tau .
\end{aligned}
$$

The periodicity condition $\mu(t+T) \equiv \mu(t)$ will be met if and only if

$$
\begin{aligned}
& {\left[\mu(0)-\frac{\alpha}{2(\epsilon \beta)^{1 / 2}}\right] \exp \left[-2(\epsilon \beta)^{1 / 2} T\right]} \\
& +2(\epsilon \beta)^{1 / 2} \exp \left[-2(\epsilon \beta)^{1 / 2} T\right] \int_{0}^{t+T} \exp \left[2(\epsilon \beta)^{1 / 2} \tau\right] \varphi(\tau) d \tau \\
& =\left[\mu(0)-\frac{\alpha}{2(\epsilon \beta)^{1 / 2}}\right] \\
& +2(\epsilon \beta)^{1 / 2} \int_{0}^{t} \exp \left[2(\epsilon \beta)^{1 / 2} \tau\right] \varphi(\tau) d \tau
\end{aligned}
$$


We now use the assumed periodicity of $\varphi(t)$ to show that $\mu(0)$ can be chosen to achieve periodicity for $\mu(t)$. We have

$$
\begin{gathered}
\int_{0}^{t+T} \exp \left[2(\epsilon \beta)^{1 / 2} \tau\right] \varphi(\tau) d \tau \\
=\int_{0}^{T} \exp \left[2(\epsilon \beta)^{1 / 2} \tau\right] \varphi(\tau) d \tau \\
\quad+\int_{T}^{t+T} \exp \left[2(\epsilon \beta)^{1 / 2} \tau\right] \varphi(\tau) d \tau \\
=\int_{0}^{T} \exp \left[2(\epsilon \beta)^{1 / 2} \tau\right] \varphi(\tau) d \tau \\
\quad+\int_{0}^{t} \exp \left[2(\epsilon \beta)^{1 / 2}(T+\xi)\right] \varphi(T+\xi) d \xi \\
=\int_{0}^{T} \exp \left[2(\epsilon \beta)^{1 / 2} \tau\right] \varphi(\tau) d \tau \\
+\exp \left[2(\epsilon \beta)^{1 / 2} T\right] \int_{0}^{t} \exp \left[2(\epsilon \beta)^{1 / 2} \xi\right] \varphi(\xi) d \xi
\end{gathered}
$$

so the periodicity condition $\mu(t+T) \equiv \mu(t)$ will be met if and only if

$$
\begin{aligned}
& {\left[\mu(0)-\frac{\alpha}{2(\epsilon \beta)^{1 / 2}}\right] \exp \left[-2(\epsilon \beta)^{1 / 2} T\right]} \\
& +2(\epsilon \beta)^{1 / 2} \exp \left[-2(\epsilon \beta)^{1 / 2} T\right] \int_{0}^{T} \exp \left[2(\epsilon \beta)^{1 / 2} \tau\right] \varphi(\tau) d \tau \\
& \quad=\left[\mu(0)-\frac{\alpha}{2(\epsilon \beta)^{1 / 2}}\right]
\end{aligned}
$$

and we arrive at the requirement that

$$
\begin{aligned}
& {\left[\mu(0)-\frac{\alpha}{2(\epsilon \beta)^{1 / 2}}\right]} \\
& \quad=\frac{2(\epsilon \beta)^{1 / 2}}{\exp \left[2(\epsilon \beta)^{1 / 2} T\right]-1} \int_{0}^{T} \exp \left[2(\epsilon \beta)^{1 / 2} \tau\right] \varphi(\tau) d \tau
\end{aligned}
$$

Hence we have determined a unique periodic solution for $\mu(t)$, namely

$$
\begin{aligned}
\mu(t) & =\frac{2(\epsilon \beta)^{1 / 2} \exp \left[-2(\epsilon \beta)^{1 / 2} t\right]}{\exp \left[2(\epsilon \beta)^{1 / 2} T\right]-1} \int_{0}^{T} \exp \left[2(\epsilon \beta)^{1 / 2} \tau\right] \varphi(\tau) d \tau \\
& +2(\epsilon \beta)^{1 / 2} \exp \left[-2(\epsilon \beta)^{1 / 2} t\right] \int_{0}^{t} \exp \left[2(\epsilon \beta)^{1 / 2} \tau\right] \varphi(\tau) d \tau \\
& +\frac{\alpha}{2(\epsilon \beta)^{1 / 2}}
\end{aligned}
$$

As a check on our algebra, even though we know what $\bar{\mu}$ needs to be, we evaluate it by direct integration of the solution that we have found. Observing that

$$
\begin{array}{rl}
\frac{1}{T} \int_{0}^{T} & 2(\epsilon \beta)^{1 / 2} \exp \left[-2(\epsilon \beta)^{1 / 2} t\right] \\
& =-\frac{1}{T}\left\{\exp \left[-2(\epsilon \beta)^{1 / 2} T\right]-1\right\} \\
& =\frac{1}{T} \exp \left[-2(\epsilon \beta)^{1 / 2} T\right]\left\{\exp \left[2(\epsilon \beta)^{1 / 2} T\right]-1\right\},
\end{array}
$$

we see that

$$
\begin{aligned}
\bar{\mu}= & \frac{1}{T} \int_{0}^{T} \mu(t) d t \\
= & \frac{\alpha}{2(\epsilon \beta)^{1 / 2}} \\
& +\frac{1}{T} \exp \left[-2(\epsilon \beta)^{1 / 2} T\right] \int_{0}^{T} \exp \left[2(\epsilon \beta)^{1 / 2} \tau\right] \varphi(\tau) d \tau \\
& +\frac{1}{T} \int_{0}^{T} 2(\epsilon \beta)^{1 / 2} \exp \left[-2(\epsilon \beta)^{1 / 2} t\right] \\
& \quad \times \int_{0}^{t} \exp \left[2(\epsilon \beta)^{1 / 2} \tau\right] \varphi(\tau) d \tau d t
\end{aligned}
$$

If we interchange orders of integration in the double integral term, it becomes

$$
\begin{aligned}
& \frac{1}{T} \int_{0}^{T} \exp \left[2(\epsilon \beta)^{1 / 2} \tau\right] \varphi(\tau) \\
& \quad \times \int_{\tau}^{T} 2(\epsilon \beta)^{1 / 2} \exp \left[-2(\epsilon \beta)^{1 / 2} t\right] d t d \tau \\
& \quad=\frac{1}{T} \int_{0}^{T} \exp \left[2(\epsilon \beta)^{1 / 2} \tau\right] \varphi(\tau)\left\{\exp \left[-2(\epsilon \beta)^{1 / 2} \tau\right]\right. \\
& \left.\quad-\exp \left[-2(\epsilon \beta)^{1 / 2} T\right]\right\} d \tau \\
& =\bar{\varphi}-\frac{\exp \left[-2(\epsilon \beta)^{1 / 2} T\right]}{T} \int_{0}^{T} \exp \left[2(\epsilon \beta)^{1 / 2} \tau\right] \varphi(\tau) d \tau
\end{aligned}
$$

Hence the mean location of the instantaneously most prevalent phenotype is

$$
\bar{\mu}=\frac{\alpha}{2(\epsilon \beta)^{1 / 2}}+\bar{\varphi}
$$

as we found earlier.

\section{A.4. A periodic solution for $\varrho(t)$}

We shall now seek a periodic solution for $\varrho(t)$, given that wherever $\mu(t)$ appears we now use the periodic solution that we have constructed. We rewrite Eq. (A.4) as

$$
-\frac{\varrho^{\prime}(t)}{\varrho(t)}+Q(t)=\kappa \varrho(t) .
$$

Let

$$
\varrho(t)=\frac{1}{\kappa r(t)},
$$

so that $\varrho^{\prime}(t) / \varrho(t)=-r^{\prime}(t) / r(t)$, giving

$$
\frac{r^{\prime}(t)}{r(t)}+Q(t)=\frac{1}{r(t)},
$$

and we now have a first-order linear ordinary differential equation:

$$
r^{\prime}(t)+Q(t) r(t)=1 .
$$

Since we were given that $\varphi(t)$ has period $T$ and we have proved that $\mu(t)$ has period $T$, we know that $Q(t)$ is also 
periodic with period $T$. Constructing an integrating factor for Eq. (A.13) in the usual way, we see that

$$
\frac{d}{d t}\left\{r(t) \exp \left[\int_{0}^{t} Q(\tau) d \tau\right]\right\}=\exp \left[\int_{0}^{t} Q(\tau) d \tau\right]
$$

and so

$$
\begin{aligned}
& r(t)=r(0) \exp \left[-\int_{0}^{t} Q(\tau) d \tau\right] \\
&+\exp \left[-\int_{0}^{t} Q(\tau) d \tau\right] \int_{0}^{t} \exp \left[\int_{0}^{\xi} Q(\tau) d \tau\right] d \xi .
\end{aligned}
$$

To have $\varrho(t+T) \equiv \varrho(t)$ we need $r(t+T) \equiv r(t)$ and this requires

$$
\begin{aligned}
& r(0) \exp \left[-\int_{0}^{t+T} Q(\tau) d \tau\right] \\
& +\exp \left[-\int_{0}^{t+T} Q(\tau) d \tau\right] \int_{0}^{t+T} \exp \left[\int_{0}^{\xi} Q(\tau) d \tau\right] d \xi \\
& =r(0) \exp \left[-\int_{0}^{t} Q(\tau) d \tau\right] \\
& +\exp \left[-\int_{0}^{t} Q(\tau) d \tau\right] \int_{0}^{t} \exp \left[\int_{0}^{\xi} Q(\tau) d \tau\right] d \xi
\end{aligned}
$$

Exploiting periodicity of $Q(t)$, we have

$$
\begin{aligned}
\int_{0}^{t+T} Q(\tau) d \tau & =\int_{0}^{T} Q(\tau) d \tau+\int_{T}^{t+T} Q(\tau) d \tau \\
& =\int_{0}^{T} Q(\tau) d \tau+\int_{0}^{t} Q(T+\tau) d \tau \\
& =\int_{0}^{T} Q(\tau) d \tau+\int_{0}^{t} Q(\tau) d \tau
\end{aligned}
$$

and so the condition for periodicity of $r(t)$ becomes

$$
\begin{aligned}
& r(0) \exp \left[-\int_{0}^{T} Q(\tau) d \tau\right] \\
& +\exp \left[-\int_{0}^{T} Q(\tau) d \tau\right] \int_{0}^{t+T} \exp \left[\int_{0}^{\xi} Q(\tau) d \tau\right] d \xi \\
& =r(0)+\int_{0}^{t} \exp \left[\int_{0}^{\xi} Q(\tau) d \tau\right] d \xi
\end{aligned}
$$

We also note that

$$
\begin{aligned}
\int_{0}^{t+T} & \exp \left[\int_{0}^{\xi} Q(\tau) d \tau\right] d \xi \\
= & \int_{0}^{T} \exp \left[\int_{0}^{\xi} Q(\tau) d \tau\right] d \xi+\int_{T}^{t+T} \exp \left[\int_{0}^{\xi} Q(\tau) d \tau\right] d \xi \\
= & \int_{0}^{T} \exp \left[\int_{0}^{\xi} Q(\tau) d \tau\right] d \xi \\
& +\int_{T}^{t+T} \exp \left[\int_{0}^{T} Q(\tau) d \tau+\int_{T}^{\xi} Q(\tau) d \tau\right] d \xi
\end{aligned}
$$

We now take $\xi=T+\zeta$ in the last integral, use periodicity of $Q(t)$ again, and deduce that

$$
\begin{aligned}
& \int_{0}^{t+T} \exp \left[\int_{0}^{\xi} Q(\tau) d \tau\right] d \xi \\
&=\int_{0}^{T} \exp \left[\int_{0}^{\xi} Q(\tau) d \tau\right] d \xi \\
& \quad+\exp \left[\int_{0}^{T} Q(\tau) d \tau\right] \int_{0}^{t} \exp \left[\int_{0}^{\xi} Q(\tau) d \tau\right] d \xi
\end{aligned}
$$

It can now be seen that all $t$-dependence cancels in Eq. (A.15) and the periodicity condition reduces to

$$
\begin{aligned}
& r(0) \exp \left[-\int_{0}^{T} Q(\tau) d \tau\right] \\
& +\exp \left[-\int_{0}^{T} Q(\tau) d \tau\right] \int_{0}^{T} \exp \left[\int_{0}^{\xi} Q(\tau) d \tau\right] d \xi=r(0)
\end{aligned}
$$

and this determines $r(0)$ uniquely:

$$
r(0)=\frac{\int_{0}^{T} \exp \left[\int_{0}^{\xi} Q(\tau) d \tau\right] d \xi}{\exp \left[\int_{0}^{T} Q(\tau) d \tau\right]-1} .
$$

We can now recover the corresponding solution for $\varrho(t)$, using Eqs (A.12) and (A.14):

$$
\varrho(t)=\frac{\kappa^{-1} \exp \left[\int_{0}^{t} Q(\tau) d \tau\right]}{r(0)+\int_{0}^{t} \exp \left[\int_{0}^{\xi} Q(\tau) d \tau\right] d \xi}
$$

where $r(0)$ is given by Eq. (A.16).

As a check on our analysis we can evaluate the mean population $\bar{\varrho}$ by integrating the solution, and compare it with what we found by direct integration of the original differential equation. We find that

$$
\begin{aligned}
T \bar{\varrho} & =\int_{0}^{T} \varrho(t) d t \\
& =\kappa^{-1} \log \left\{1+\frac{1}{r(0)} \int_{0}^{T} \exp \left[\int_{0}^{\xi} Q(\tau) d \tau\right] d \xi\right\}
\end{aligned}
$$

and inserting the value of $r(0)$ that we have determined, we find that

$$
T \bar{\varrho}=\frac{1}{\kappa} \int_{0}^{T} Q(\tau) d \tau
$$

so we recover the previously determined mean population $\bar{\varrho}=\bar{Q} / \kappa$.

We now address the parameter restrictions that may be needed for the analysis to be valid. To obtain a nonnegative, finite total population $\varrho(t)$, we require $r(t)>0$ for all time. Since the solution we have constructed is periodic, we need only pursue this matter for $t \geq 0$. If we examine Eq. (A.14), we see that $r(0)>0$ for all $t \geq 0$ if 
and only if $r(0)>0$, and this requires the denominator in Eq. (A.16) to be strictly positive, which in turn requires that

$$
\int_{0}^{T} Q(t) d t=T \bar{Q}>0 .
$$

\section{A.5. When can $\varrho(t)$ oscillate faster than $\varphi(t)$ ?}

The proof of periodicity of $\varrho(t)$ used only the periodicity of

$$
Q(t)=\gamma-\epsilon[\varphi(t)-\mu(t)]^{2}-(\epsilon \beta)^{1 / 2}
$$

and $\varrho(t)$ has the same period. Since we had established earlier that there is a valid Gaussian solution $\mathcal{C}(x, t)$ for the phenotype distribution with $\mu(t+T) \equiv \mu(t)$ when $\varphi(t+$ $T) \equiv \varphi(t)$, it followed that $Q(t+T) \equiv Q(t)$. However, since it is $[\varphi(t)-\mu(t)]^{2}$, rather than $\varphi(t)-\mu(t)$, that appears in the formula defining $Q(t)$, we see that a sufficient condition for $Q(t+T / 2) \equiv Q(t)$, and hence for $\varrho(t+T / 2) \equiv \varrho(t)$, is

$$
\varphi(t+T / 2)-\mu(t+T / 2)=-[\varphi(t)-\mu(t)]
$$

and invoking the assumption

$$
\varphi(t+T / 2)=-\varphi(t)
$$

the sufficient condition is that

$$
\mu(t+T / 2)+\mu(t) \equiv 0 .
$$

Using assumption (A.18) we see that

$$
\begin{aligned}
\int_{0}^{T} \exp \left[2(\epsilon \beta)^{1 / 2} \tau\right] \varphi(\tau) d \tau & \\
= & \int_{0}^{T / 2} \exp \left[2(\epsilon \beta)^{1 / 2} \tau\right] \varphi(\tau) d \tau \\
& \quad+\int_{T / 2}^{T} \exp \left[2(\epsilon \beta)^{1 / 2} \tau\right] \varphi(\tau) d \tau \\
= & \int_{0}^{T / 2} \exp \left[2(\epsilon \beta)^{1 / 2} \tau\right] \varphi(\tau) d \tau \\
& \quad+\int_{0}^{T / 2} \exp \left[2(\epsilon \beta)^{1 / 2}(\tau+T / 2)\right] \varphi(\tau+T / 2) d \tau \\
= & -\left\{\exp \left[(\epsilon \beta)^{1 / 2} T\right]-1\right\} \int_{0}^{T / 2} \exp \left[2(\epsilon \beta)^{1 / 2} \tau\right] \varphi(\tau) d \tau .
\end{aligned}
$$

Setting $\alpha=0$ in our general solution for $\mu(t)$ we now have

$\mu(t)$

$$
\begin{aligned}
= & \frac{2(\epsilon \beta)^{1 / 2} \exp \left[-2(\epsilon \beta)^{1 / 2} t\right]}{\exp \left[2(\epsilon \beta)^{1 / 2} T\right]-1} \int_{0}^{T} \exp \left[2(\epsilon \beta)^{1 / 2} \tau\right] \varphi(\tau) d \tau \\
& +2(\epsilon \beta)^{1 / 2} \exp \left[-2(\epsilon \beta)^{1 / 2} t\right] \int_{0}^{t} \exp \left[2(\epsilon \beta)^{1 / 2} \tau\right] \varphi(\tau) d \tau \\
= & -\frac{2(\epsilon \beta)^{1 / 2} \exp \left[-2(\epsilon \beta)^{1 / 2} t\right]}{\exp \left[(\epsilon \beta)^{1 / 2} T\right]+1} \int_{0}^{T / 2} \exp \left[2(\epsilon \beta)^{1 / 2} \tau\right] \varphi(\tau) d \tau \\
& +2(\epsilon \beta)^{1 / 2} \exp \left[-2(\epsilon \beta)^{1 / 2} t\right] \int_{0}^{t} \exp \left[2(\epsilon \beta)^{1 / 2} \tau\right] \varphi(\tau) d \tau .
\end{aligned}
$$

It now follows that

$$
\begin{aligned}
& \frac{\mu(t+T / 2)+\mu(t)}{2(\epsilon \beta)^{1 / 2} \exp \left[-2(\epsilon \beta)^{1 / 2} t\right]} \\
& =-\exp \left[-(\epsilon \beta)^{1 / 2} T\right] \int_{0}^{T / 2} \exp \left[2(\epsilon \beta)^{1 / 2} \tau\right] \varphi(\tau) d \tau \\
& \quad+\exp \left[-(\epsilon \beta)^{1 / 2} T\right] \int_{0}^{t+T / 2} \exp \left[2(\epsilon \beta)^{1 / 2} \tau\right] \varphi(\tau) d \tau \\
& \quad+\int_{0}^{t} \exp \left[2(\epsilon \beta)^{1 / 2} \tau\right] \varphi(\tau) d \tau
\end{aligned}
$$

Next we exploit assumption (A.18) again:

$$
\begin{aligned}
\int_{0}^{t+T / 2} & \exp \left[2(\epsilon \beta)^{1 / 2} \tau\right] \varphi(\tau) d \tau \\
= & \int_{0}^{T / 2} \exp \left[2(\epsilon \beta)^{1 / 2} \tau\right] \varphi(\tau) d \tau \\
& +\int_{T / 2}^{t+T / 2} \exp \left[2(\epsilon \beta)^{1 / 2} \tau\right] \varphi(\tau) d \tau \\
= & \int_{0}^{T / 2} \exp \left[2(\epsilon \beta)^{1 / 2} \tau\right] \varphi(\tau) d \tau \\
& \quad+\int_{0}^{t} \exp \left[2(\epsilon \beta)^{1 / 2}(\tau+T / 2)\right] \varphi(\tau+T / 2) d \tau \\
= & \int_{0}^{T / 2} \exp \left[2(\epsilon \beta)^{1 / 2} \tau\right] \varphi(\tau) d \tau \\
& \quad-\exp \left[(\epsilon \beta)^{1 / 2} T\right] \int_{0}^{t} \exp \left[2(\epsilon \beta)^{1 / 2} \tau\right] \varphi(\tau) d \tau
\end{aligned}
$$

and we now see that

$$
\frac{\mu(t+T / 2)+\mu(t)}{2(\epsilon \beta)^{1 / 2} \exp \left[-2(\epsilon \beta)^{1 / 2} t\right]} \equiv 0
$$

Thus the sufficient condition (A.19) is met if the assumptions $\alpha=0$ and $\varphi(t+T / 2)=-\varphi(t)$ are made.

\section{B. Details of numerical solutions}

We describe here the procedure for constructing numerical solutions of the mathematical problem defined by endowing (2.1)-(2.2) with the initial condition

$$
c(x, 0)=C_{0} \mathbb{1}_{(-L ; L)}(x), \quad C_{0}=40,
$$

and the Dirichlet boundary conditions

$$
c(-L, \cdot)=c(L, \cdot)=0 .
$$

The above conditions satisfy assumption (2.3) and have a similar effect to assumption (2.4), replacing a significant rate of death at all sufficiently large $|x|$ by certain death at one sufficiently large value of $|x|$.

We fix a time step $\Delta t$ and set $t_{k}=k \Delta t$. The method is based on a time splitting scheme between the conservative part and the reaction term, that is, the approximation 
$c^{k+1}$ of $c\left(t_{k+1}\right)$ is computed from the approximation $c^{k}$ of $c\left(t_{k}\right)$ in two steps:

$$
c^{k+1 / 2}=c^{k}-\Delta t\left(\alpha \frac{\partial c^{k}}{\partial x}-\beta \frac{\partial^{2} c^{k}}{\partial x^{2}}\right)
$$

and

$$
c^{k+1}=c^{k+1 / 2}+\Delta t c^{k+1 / 2} R\left(x, \varphi^{k}, \varrho^{k}\right),
$$

where $\varphi^{k}$ is the approximation of $\varphi\left(t_{k}\right)$ and $\varrho^{k}$ is the integral of $c^{k}$. We next turn to the space discretisation and we use a uniform grid with $N$ points on the interval $[-L, L]$, with $\Delta x=2 L / N$ the space step. We approximate $c^{k}\left(x_{i}\right)$ and $R\left(x_{i}, \varphi^{k}, \varrho^{k}\right)$ by discrete values $c_{i}^{k}$ and $R_{i}\left(\varphi^{k}, \varrho^{k}\right)$, and we recover $\varrho^{k}$ through numerical integration. We solve Eq.(B.1) by using a second-order upwind scheme for the advection term and a three points explicit scheme for the diffusion term. Since we choose $\beta$ small, the explicit scheme is not penalising in terms of computational time. For the reaction term, we use an implicit-explicit finite difference scheme [19], that is, we compute $c_{i}^{k+1}$ as

$$
c_{i}^{k+1}=c_{i}^{k+1 / 2} \frac{1+\Delta t R_{i}\left(\varphi^{k}, \varrho^{k}\right)_{+}}{1+\Delta t R_{i}\left(\varphi^{k}, \varrho^{k}\right)_{-}} .
$$

Numerical computations are performed in MATLAB. We select a uniform discretisation consisting of 600 points on the interval $[-L, L]$ with $L=2$ as the spatial domain, and the interval $[0,20]$ as the time domain (time step $\Delta t=$ $10^{-5}$ ). Unless otherwise stated, we set $T=5, \gamma=200$, $\epsilon=30, \kappa=1$ and

$$
\varphi(t):=\sin (\omega t), \quad \omega=\frac{2 \pi}{5} .
$$

The values of the other parameters of the model are listed below.

Figure 1: $\alpha=0, \beta=0.5$.

Figure 2: $\alpha=0, \beta=0.5$ (solid lines); $\alpha=1, \beta=0.5$ (dashed lines); $\alpha=2, \beta=0.5$ (dotted lines).

Figure 3: $\alpha=0.1, \beta \in[0.01 ; 1], \epsilon \in[0.01 ; 100]$.

Figure $4(\mathrm{~A}): \alpha \in[0.01 ; 10], \beta=0.5, \epsilon \in[1 ; 100]$.

Figure 4(B): $\alpha \in[0.01 ; 10], \beta \in[0.01 ; 1], \epsilon=30$.

Figure 5: $\alpha=0.1, \beta \in[0.01 ; 1], \gamma=30, \epsilon=30, T \in$ $[0.01 ; 5]$.

\section{References}

[1] Acar, M., Mettetal, J.T., van Oudenaarden, A. 2008. Stochastic switching as a survival strategy in fluctuating environments. Nat. Genet. 40, 471-475.

[2] Avery, S.V. 2006. Microbial cell individuality and the underlying sources of heterogeneity. Nat. Rev. Microbiol. 4, 577-587.
[3] Casadesus, J., Low, D. 2006. Epigenetic gene regulation in the bacterial world. Microbiol. Mol. Biol. Rev. 70, 830-856.

[4] Dubnau, D., Losick, R. 2006. Bistability in bacteria. Mol. Microbiol. 61, 564-572.

[5] Gander, M.J., Mazza, C., Rummler, H. 2007. Stochastic gene expression in switching environments. J. Math. Biol. 55, 259294.

[6] Kussell, E., Kishony, R., Balaban, N.Q., Leibler, S. 2005. Bacterial persistence: a model of survival in changing environments. Genetics 169, 1807-1814.

[7] Kussell, E., Leibler, S. 2005. Phenotypic diversity, population growth, and information in fluctuating environments. Science 309, 2075-2078.

[8] Lachmann, M., Jablonka, E. 1996. The inheritance of phenotypes: an adaptation to fluctuating environments. J. Theor. Biol. 181, 1-9.

[9] Thattai, M., and van Oudenaarden, A. 2004. Stochastic gene expression in fluctuating environments. Genetics 167, 523-530.

[10] Wolf, D.M., Vazirani, V.V., Arkin, A.P. 2005. Diversity in times of adversity: probabilistic strategies in microbial survival games. J. Theor. Biol. 234, 227-253.

[11] Champagnat, N., Ferrière, R., Méléard, S. 2006. Unifying evolutionary dynamics: From individual stochastic processes to macroscopic models. Theor. Popul. Biol. 69, 297-321.

[12] Champagnat, N., Ferrière, R., Ben Arous, G. 2001. The canonical equation of adaptive dynamics: A mathematical view. Selection 2, 73-83.

[13] Bouin, E., Calvez, V. 2014. Travelling waves for the cane toads equation with bounded traits. Nonlinearity 27, 2233, doi:10.1088/0951-7715/27/9/2233.

[14] Bouin, E., Calvez, V., Meunier, N., Mirrahimi, S., Perthame, B., Raoul, G., et al. 2012. Invasion fronts with variable motility: phenotype selection, spatial sorting and wave acceleration. Comptes Rendus Math. 350, 761-766

[15] Chisholm, R.H., Lorenzi, T., Lorz, A., Larsen, A.K., Neves de Almeida, L., Escargueil, A. et al. 2015. Emergence of drug tolerance in cancer cell populations: an evolutionary outcome of selection, non-genetic instability and stress-induced adaptation. Cancer Res. 75, 930-939.

[16] Delitala, M., Dianzani, U., Lorenzi, T., Melensi, M. 2013. A mathematical model for immune and autoimmune response mediated by T-cells. Comp. Math. Appl. 66, 1010-1023.

[17] Delitala, M., Lorenzi, T. 2012. A mathematical model for the dynamics of cancer hepatocytes under therapeutic actions. J. Theor. Biol. 297, 88-102.

[18] Lavi, O., Greene, J., Levy, D., Gottesman, M., Simplifying the complexity of resistance heterogeneity in metastatic cancer. 2014. Trends Mol. Med. 20, 129-136.

[19] Lorz, A., Lorenzi, T., Hochberg, M.E., Clairambault, J., Perthame, B. 2013. Populational adaptive evolution, chemotherapeutic resistance and multiple anticancer therapies. ESAIMMath. Model. Num. 47, 377-399.

[20] Stiehl, T., Baran, N., Ho, A.D., Marciniak-Czochra, A. 2014. Clonal selection and therapy resistance in acute leukaemias: mathematical modelling explains different proliferation patterns at diagnosis and relapse. J. R. Soc. Interface. 11(94):20140079.

[21] Brock, A., Chang, H., Huang, S. 2009. Non-genetic heterogeneity - a mutation-independent driving force for the somatic evolution of tumours. Nat. Rev. Genet. 10, 336-342.

[22] Sharma, S.V., Lee, D.Y., Li, B., Quinlan, M.P., Takahashi, F., Maheswaran, S., et al. 2010. A chromatin-mediated reversible drug-tolerant state in cancer cell subpopulations. Cell, 141, 6980 .

[23] Gupta, P.B., Fillmore, C.M., Jiang, G., Shapira, S.D., Tao, K., Kuperwasser, C., et al. 2011. Stochastic state transitions give rise to phenotypic equilibrium in populations of cancer cells. Cell, 146, 633-644.

[24] Pisco, A.O., Brock, A., Zhou, J., Moor, A., Mojtahedi, M., Jackson, D., et al. 2013. Non-Darwinian dynamics in therapyinduced cancer drug resistance. Nat. Commun. 4, 3467.

[25] Becker, C., Hagmann, J., Müller, J., Koenig, D., Stegle, O., 
Borgwardt, K., et al. 2011. Spontaneous epigenetic variation in the arabidopsis thaliana methylome. Nature 480, 245-249.

[26] Lorz, A., Mirrahimi, S., Perthame, B. 2011. Dirac mass dynamics in a multidimensional nonlocal parabolic equation. Commun. Part. Differ. Equ. 36, 1071-1098.

[27] Mirrahimi, S., Perthame, B., Souganidis, P.E. 2015. Time fluctuations in a population model of adaptative dynamics. Ann. I. H. Poincaré-AN 32, 41-58.

[28] Perthame, B., Barles, G. 2008. Dirac concentrations in LotkaVolterra parabolic PDEs. Indiana Univ. Math. J. 57, 3275-3301.

[29] Calsina, À., Cuadrado, S., Desvillettes, L., Raoul G. 2013. Asymptotics of steady states of a selection-mutation equation for small mutation rate. P. Roy. Soc. Edinb. A. 143, 1123-1146.

[30] Desvillettes L., Jabin, P.-E., Mischler, S., Raoul, G. 2008. On selection dynamics for continuous structured populations, Commun. Math. Sci. 6, 729-747.

[31] Raoul, G. 2011. Long time evolution of populations under selection and vanishing mutations. Acta Appl. Math. 114, 1-14.

[32] Ashcroft, P., Altrock, P.M., Galla, T. 2014 Fixation in finite populations evolving in fluctuating environments. J. R. Soc. Interface 11:20140663.

[33] Arthur, W., Farrow, M. 1999. The pattern of variation in centipede segment number as an example of developmental constraint in evolution. J. Theor. Biol. 200, 183-191.

[34] Donoghue, M.J., Ree, M. 2000. Homoplasy and developmental constraint: a model and an example from plants. Am. Zool. 40, 759-769.

[35] Laland, K., Uller, T., Feldman, M., Sterelny, K., Müller, G.B., Moczek, A., et al. 2014. Does evolutionary theory need a rethink? Nature 514, 161-164.

[36] Wallace, A. 2002. The emerging conceptual framework of evolutionary developmental biology. Nature. 415, 757-764.

37] Chisholm, R.H., Lorenzi, T., Hughes, B.D. 2015. The adaptive value of epigenetic variation in colonising asexual populations. Preprint.

[38] Servedio, M.R., Brandvain, Y., Dhole, S., Fitzpatrick, C.L., Goldberg, E.E., Stern, C.A., et al. 2014. Not just a theory-the utility of mathematical models in evolutionary biology. PLoS Biol. 12(12): e1002017.

[39] Klironomos, J.N., Allen, M.F., Matthias, C.R., Piotrowski, J., Makvandi-Nejad, S., Wolfe, B.E., et al. 2005. Abrupt rise in atmospheric $\mathrm{CO} 2$ overestimates community response in a model plant-soil system. Nature 433, 621-624.

[40] Yampolsky, L.Y., Stoltzfus, A. 2001. Bias in the introduction of variation as an orienting factor in evolution. Evol. Dev. 3, 73-83.

[41] Chevin, L.-M., Gallet, R., Gomulkiewicz, R., Holt, R.D., Fellous, S. 2013. Phenotypic plasticity in evolutionary rescue experiments. Philos. Trans. R. Soc. B Biol. Sci. 368:20120089.

[42] Reed, T.E., Waples, R.S., Schindler, D.E., Hard, J.J., Kinnison, M.T. 2010. Phenotypic plasticity and population viability: the importance of environmental predictability. Proc. R Soc. B. 277, $3391-3400$. 


\section{University Library}

\section{- M M I N E R VA \\ A gateway to Melbourne's research publications}

Minerva Access is the Institutional Repository of The University of Melbourne

Author/s:

Lorenzi, T;Chisholm, RH;Desvillettes, L;Hughes, BD

Title:

Dissecting the dynamics of epigenetic changes in phenotype-structured populations exposed to fluctuating environments

Date:

2015-12-07

Citation:

Lorenzi, T., Chisholm, R. H., Desvillettes, L. \& Hughes, B. D. (2015). Dissecting the dynamics of epigenetic changes in phenotype-structured populations exposed to fluctuating environments. JOURNAL OF THEORETICAL BIOLOGY, 386, pp.166-176. https:// doi.org/10.1016/j.jtbi.2015.08.031.

Persistent Link:

http://hdl.handle.net/11343/58816 